\title{
Spectroscopic Studies of Complex of Poly Aniline with Zinc Ion in Finger Print Region
}

\author{
Anubha Vijay Pandya \\ Prof. and Head of the Department Chemical Sciences \\ Christian Eminent College Indore
}

\begin{abstract}
Spectroscopic studies are the most advanced technique and hence has proven as powerful tool for any type of qualitative and quantitative analysis . Infrared spectroscopy is one of the most powerful analytical techniques which offers the possibility of chemical identification. This technique when coupled with intensity measurements may be used for quantitative analysis .one of the most important advantages of infrared spectroscopy over the other usual methods of structural analysis such as X-ray diffraction analysis ,electron spin resonance etc. is that it provides useful information about the structure of compounds and can solve many problems in organic chemistry and coordination chemistry .In present paper,with the help of Infrared spectroscopy conformation of polymer -metal complex formation has been assured that can help to study of polymer as conducting material to prepare polymer electrodes and polymer electrolytes .This research paper will help to prepare polymer batteries. For this purpose polymer, metal-polymer complex like Poly Aniline and Poly Aniline-Zinc complex have been tested using Potassium Bromide as reference material with the help of Infrared spectrophotometer.
\end{abstract}

Keywords - Selective absorption, IR radiations, Polymer electrolytes, polymer electrodes, conducting poly aniline.

\section{INTRODUCTION}

The technique is based on the simple fact that a chemical substance shows marked selective absorption in the infrared region. After absorption of IR radiations the molecule of a chemical substance vibrates at many rates of vibration, giving rise to close packed absorption bands called an IR absorption spectrum which may extend over wide wavelength range. Various bands will be present in IR spectrum which will correspond to the characteristic functional groups and bonds present in a chemical substance are a fingerprint for its identification.

In a complex polymer molecule ${ }^{1}$ the number of infrared transition might be expected to be too great to deal with, but fortunately this does not happen as a great, many of these are degenerate, i.e.of the same energy. The advantage of fingerprint region ${ }^{5}$ has been taken in the identification of polymers. The spectrum of the unknown sample is matched against that of an authentic sample, peak by peak, and the identity of the compound ${ }^{6}$ established.

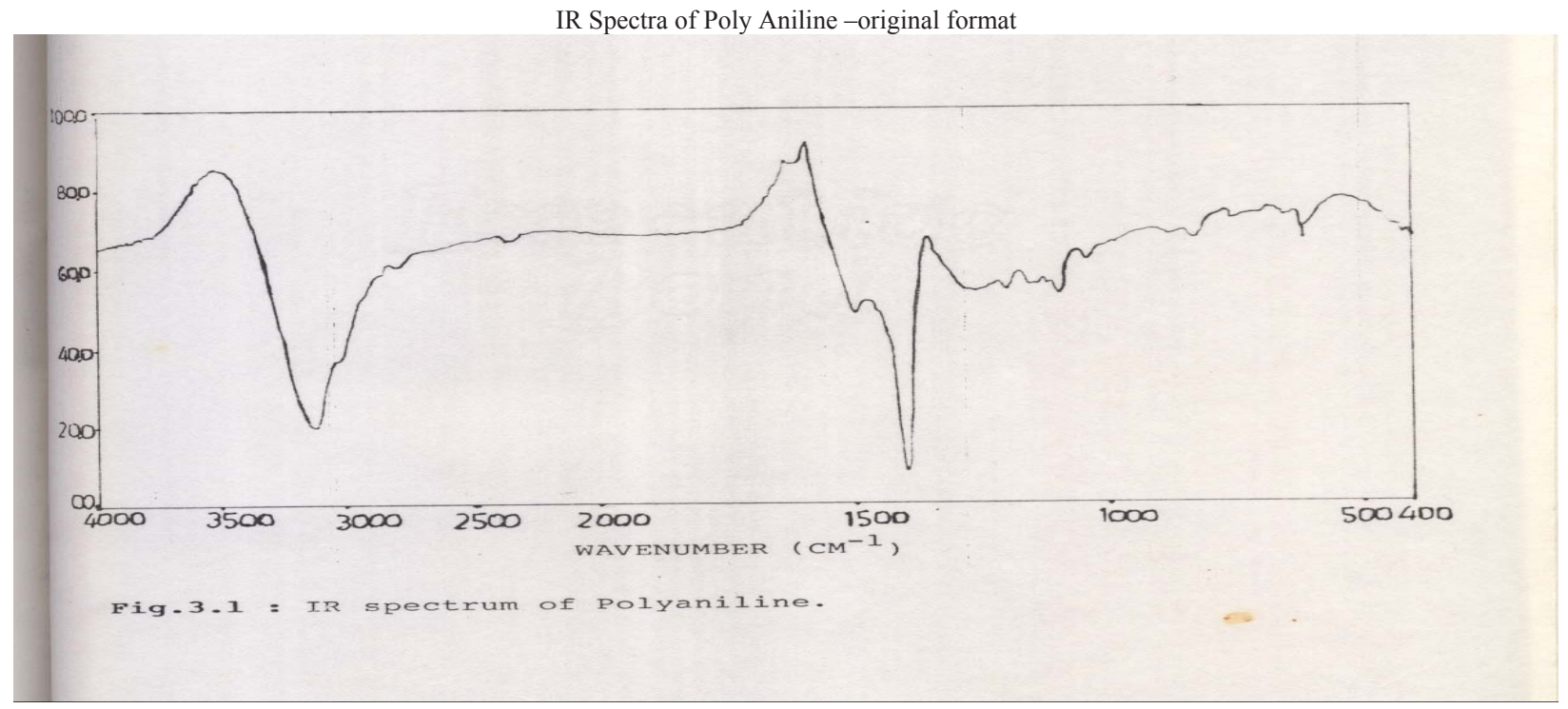




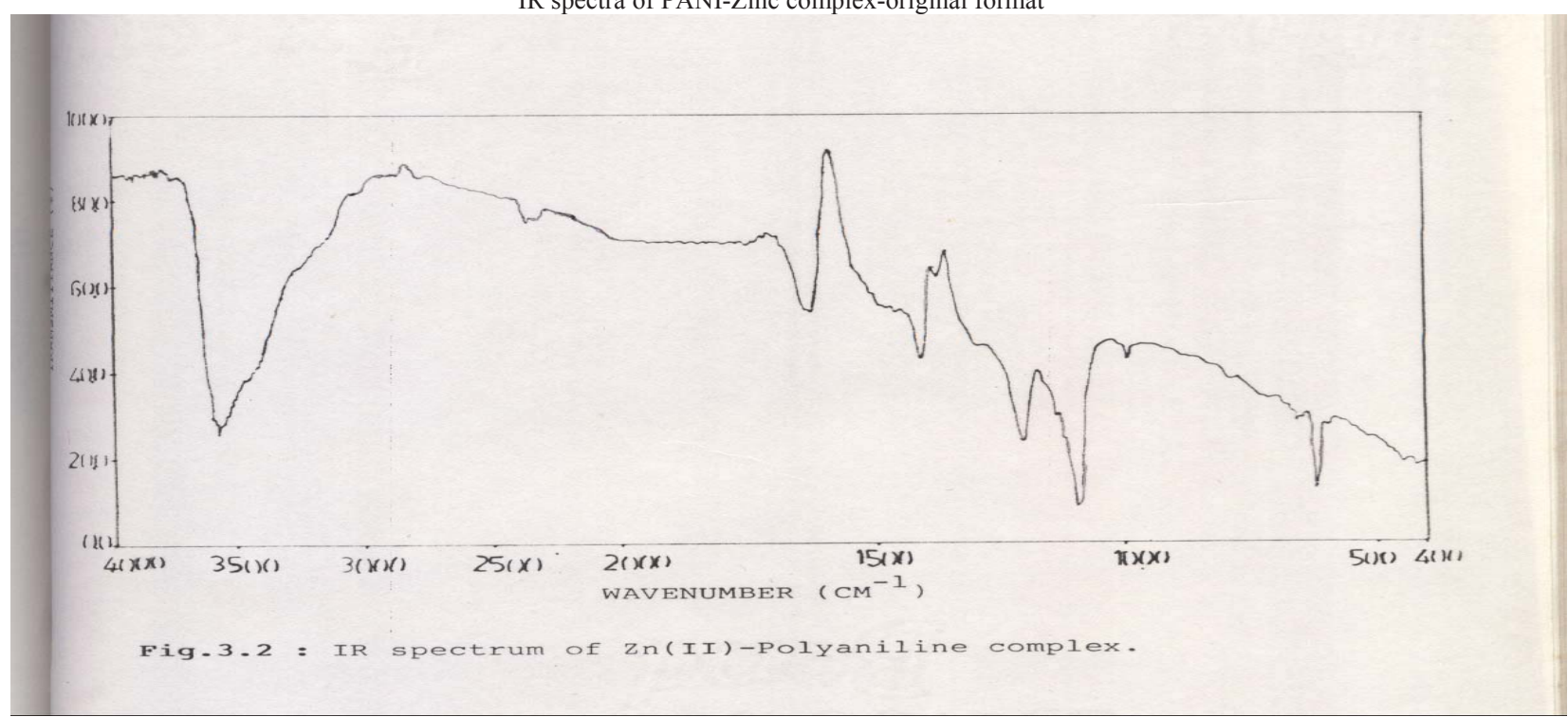

\section{Ligand}

3500 I $\mathrm{br}$

3300_I

1600

$1500 \mathrm{~s}$

Aromatic nature

$1380^{-} \mathrm{I}$ br

1360_I

$1100 \mathrm{~m}$

$780^{-} \mathrm{I}$ br

620_I

Ligand
3500 - I br
$3300 \_\mathrm{I}$
1600
$1500 \mathrm{~s}$
Aromatic nature

assignment

-NH stretching vibration

$-\mathrm{C}=\mathrm{C}$

-NH-bending

-C-N vibration

aromatic secondary

Amine

$\mathrm{CH}$ in plane stretching

$\mathrm{CH}$ bending

vibration

\section{Zn-(II)- PANI Complex}

3565

1626

(1)

S-sharp,

m-medium,

w-weak, 


\section{RESULTS AND DISCUSSION}

IR Spectra of Polyaniline and its complex with Zinc (II) metal ion has been depicted in figure . The important Infrared signal and their group assignments ${ }^{4}$ have been tabulated in table

A perusal of the figure and table reveals that the signals due to NH-stretching vibrations at $3500-3300 \mathrm{~cm}^{-1}$ and $-\mathrm{CN}$ aromatic vibration in the ligand spectra undergoes a shift in the spectrum of Zn(II)-PANI complex indicating the involvement of $-\mathrm{NH}$ nitrogen complex formation.

\section{SURVEY OF LITERATURE}

Infrared spectrum of Polymer such as PolyAniline and its $1 / 1$ blend with PAA : at $25^{\circ} \mathrm{c}$ before heating and at $25^{\circ} \mathrm{c}$ after heating to $80^{\circ} \mathrm{c}$ and other various conditions have been reported by Show-An andHsun-Tsinglee,1. They concluded that for the PANI (termed as PANI/PAA blend), the confinement of carboxylic acid group.

Ming Xiang of China studied Infrared of PVAL $(\mathrm{OH}, 47 \mathrm{~mol}) / \mathrm{PMMA}^{2}$.He concluded that relatively strong Intermolecular Hydrogen bonding interaction exists between components in the miscible blends. The curve resolving studies of FTIR spectra ${ }^{2}$ have suggested that the weakly self associated hydroxyl groups in polyvinyl acetyls are liable to form hydrogen bonds with carbonyl groups in PMMA. IR spectra of Copolymer ${ }^{7}$ and blend of bismaleimides with phenolalkyle resin (KBr)' have been reported by Liu Quingmin Xingxian ${ }^{3}$.

\section{REFERENCES}

[1] Show an chem. Macromolecule28, 2866, (1995)N.Javaid et al., "EDDEEC: Enhanced Developed Distributed Energy-Efficient Clustering for Heterogeneous Wireless Sensor Networks.", International Workshop on Body Area Sensor Networks, 2013, pp.914-919.

[2] Leo-wang, International symposia 122, 1990

[3] Ming Xiang, China.IUPAC International symposia, 7-9 nov. 76, 1990

[4] LHL-7-9 IUPAC International symposia nov227-1990

[5] A.Gadisa, Tvingstedt, S.Admassie, L.Lindeel, X.Cripsin, M.R.Anderson, W.R.Salanech, O. Inganas, Synth .met, 2006,156, 1102.

[6] K.Yoshino, Synth. Met, 1989, 28, C669.

[7] J.C.Chiang and A.G.Mac Diarmid, Synth. Met, 1986, 13, 193. 